\title{
Role of intrapulmonary lymph node retrieval for pathological examination in resectable non-small cell lung cancer
}

\author{
Apichat Tantraworasin ${ }^{1}$, Emanuela Taioli ${ }^{2}$, Sophon Siwachat ${ }^{1}$, Somcharoen Saeteng $^{1}$ \\ ${ }^{1}$ Department of Surgery, Faculty of Medicine, Chiang Mai University, Chiang Mai, Thailand; ${ }^{2}$ Population Health Science and Policy and Institute \\ for Translational Epidemiology, Icahn School of Medicine at Mount Sinai, New York, USA \\ Correspondence to: Asist. Prof. Apichat Tantraworasin, MD, PhD. General Thoracic Surgery Unit, Department of Surgery, Faculty of Medicine, \\ Chiang Mai University, Chiang Mai 50200, Thailand. Email: Apichat.t@cmu.ac.th or ohm_med@hotmail.com. \\ Provenance: This is an invited Editorial commissioned by the Section Editor Laura Chiara Guglielmetti (Cantonal Hospital Winterthur, Kantonsspital \\ Winterthur, Winterthur, Switzerland). \\ Comment on: Wang X, Yan S, Lv C, et al. Impact of Omission of Intrapulmonary Lymph Node Retrieval on Outcome Evaluation of Lung Cancer \\ Patients Without Lymph Node Metastasis: A Propensity Score Matching Analysis. Clin Lung Cancer 2017. [Epub ahead of print].
}

Submitted Oct 02, 2017. Accepted for publication Oct 16, 2017.

doi: $10.21037 /$ jtd.2017.10.118

View this article at: http://dx.doi.org/10.21037/jtd.2017.10.118

Lymph node (LN) metastasis is a prognostic factor for tumor recurrence and overall survival (OS) in resectable non-small cell lung cancer (NSCLC) patients $(1,2)$. It also affects the decision of prescribing adjuvant or neo-adjuvant systemic chemotherapy or radiotherapy. Current studies and guidelines suggest that systematic LN dissection or sampling should be performed in resectable NSCLC $(3,4)$. It is indicated that mediastinal LN (stations $2-9$ or N2) $(5,6)$ should be dissected when performing curative pulmonary resection in order to accurately determine tumor stage. For intrapulmonary LN or N1, stations 10-12 are usually dissected because they are easily identified and collected; however, the dissection of stations $13-14$ is quite difficult and requires adequate training (7). A recent study showed that standard pathology practice frequently omitted to examine $60 \%$ of intrapulmonary LNs in $90 \%$ of lobectomy specimens, and found unexpected LN metastases in $12 \%$ of reported node-negative patients (8). Nodal metastases affect the classification of the case into $\mathrm{N} 2$ or N1 disease, which in turn affects treatment strategies, tumor recurrence, and survival. It often happens that in N1 disease stations 13 and 14 are neglected, leading to disease under staging, and lack of prescription of adjuvant therapy. These may contribute to the observation that some of patients with N0 disease develop early tumor recurrence or distant metastases (8).

In the paper entitled "Impact of Omission of Intrapulmonary Lymph Node Retrieval on Outcome Evaluation of Lung Cancer
Patients Without Lymph Node Metastasis: A Propensity Score Matching Analysis" (9), Wang and colleagues retrospectively reviewed 442 clinical early-stage NSCLC who underwent pulmonary resection with systematic $\mathrm{LN}$ dissection, pathologically confirmed complete resection and pathologic N0 disease, to test the association between omitting the retrieval of $\mathrm{LN}$ from stations 13 and 14 and outcomes [5year OS and disease free survival (DFS)]. Patients were divided into two groups; the study group (patients whom LN stations 10 to 14 were collected during surgery) and the control group (patients whom LN stations 10 to 12 were retrieved during surgery). They used a matched propensity score analysis to adjust for confounders by indication. The results show that the 5 -years OS $(89 \% \pm 3 \%$ versus $77 \% \pm 4 \%, \mathrm{P}=0.027)$ and 5 -year DFS $(81 \% \pm 4 \%$ versus $67 \% \pm 4 \%, \mathrm{P}=0.021$ ) was significantly better when the surgeon collected $\mathrm{LN}$ stations 10 to 14 . The multivariable analysis demonstrated that intrapulmonary LN 13 to 14 retrieval had a significant impact on OS [hazard ratio (HR), 0.518; 95\% confidence interval (CI), 0.298-0.898] and DFS (HR, 0.590; 95\% CI, 0.387-0.901). Although the metastatic status of LN stations 13 and 14 in the control group was unknown, it is possible that the omission of the intrapulmonary $\mathrm{LN}$ collection might lead to an inferior oncologic outcome. Although intrapulmonary LN dissection was performed by surgeons only in this study, we think that pathologist could play a vital role by dissecting 
LNs from lung specimen. The authors were also concerned about this issue and suggested that both surgeons and pathologists handle the intrapulmonary LN dissection after adequate training, and the time required for the procedure was less than 10 minutes (10).

There are some previous studies dealing with intrapulmonary LN examination in NSCLC. Ramirez and colleagues (8) performed a case-control study of a special pathologic examination protocol using thin gross dissection with retrieval and microscopic examination of all LN-like material on remnant NSCLC resection specimens after routine pathologic examination. They retrieved additional LNs in $66(90 \%)$ of 73 patients and found metastasis in 56 (11\%) of 514 retrieved LNs from $27 \%$ of all patients. There were unexpected LN metastases in $6(12 \%)$ of the 50 nodenegative patients. The pathologic stage of $8(11 \%)$ of the 73 patients changed after this special pathologic examination. The time range used for the dissection of the remnant lung specimens was 10-30 minutes. The authors suggested to develop a protocol for dissection and examination of lung resection specimens, performed by surgeons or pathologists to improve $\mathrm{LN}$ yield.

Smeltzer and colleagues (11) conducted a prospective cohort study of 110 resectable NSCLC patients to evaluate undissected intrapulmonary LNs from lung resection specimens. They applied a thin-section gross re-dissection protocol to retrieve all material that appeared to be LNs and processed it for histologic examination. They found 25 patients $(23 \%)$ with additional LNs with metastases. Of these 25 patients, 6 were pN0 after routine pathologic examination. Eleven of the 25 patients had more than 2 additional LNs with metastases. In summary, 6 patients were up-staged (from pN0 to $\mathrm{pN} 1$ ) after re-dissection of the discarded lung resection specimen. Patients with at least one missed metastatic LN had increased risk of death (adjusted HR, 1.4; 95\% CI, 0.6-3.7).

Osarogiagbon and colleagues (7) retrospectively reviewed the lung resection specimens over a period of 15 months before and 15 months after the training of the pathologist's assistants on the novel dissection protocol, which included recording the dissection start and end time, and the details of the intrapulmonary LN dissection method. Although there was no difference in nodal stage distribution after using this protocol, they found that the median number of intrapulmonary LNs retrieved increase from 2 to 5 nodes $(\mathrm{P}<0.001)$ and the $75^{\text {th }}$ to $100^{\text {th }}$ percentile range of detected intrapulmonary LN metastases increased from $0-5$ to $0-17$ $(\mathrm{P}=0.0003)$. Osarogiagbon and colleagues (12) re-analyzed the LN dissection arm of the American College of Surgeons Oncology Group Z0030 trial (13) to explore the survival impact in the number of LN dissected in NSCLC patients who underwent pulmonary resection for $\mathrm{N} 0$ or nonhilar $\mathrm{N} 1, \mathrm{~T} 1$, or $\mathrm{T} 2$ disease. The $\mathrm{pN}$ category was associated with an increased number of examined intrapulmonary LNs. Patients with $\mathrm{pN} 1$ had more non-hilar N1 nodes than those with $\mathrm{pN} 0$. Patients with $\mathrm{pN} 0$ had better survival with the examination of more $\mathrm{N} 1$ nodes. The more intrapulmonary LNs were dissected, the higher was the chance to have a complete nodal staging, and this may achieve the better survival.

In conclusion, for resectable NSCLC patients, adequate intrapulmonary LN dissection should be performed during pulmonary resection or pathologic examination in gross specimen to achieve accurate pathological staging, since this has an impact on adjuvant treatment and survival. Both the surgeons and the pathologists have to consider this and should be encouraged to perform the dissection of intrapulmonary LNs in routine clinical practice of lung cancer surgery.

\section{Acknowledgements}

None.

\section{Footnote}

Conflicts of Interest: The authors have no conflicts of interest to declare.

\section{References}

1. Tantraworasin A, Saeteng S, Siwachat S, et al. Impact of lymph node management on resectable non-small cell lung cancer patients. J Thorac Dis 2017;9:666-74.

2. Tamura M, Matsumoto I, Saito D, et al. Lymph node ratio as a prognostic factor in patients with pathological N2 non-small cell lung cancer. World J Surg Oncol 2016;14:295.

3. Howington JA, Blum MG, Chang AC, et al. Treatment of stage I and II non-small cell lung cancer: Diagnosis and management of lung cancer, 3 rd ed: American College of Chest Physicians evidence-based clinical practice guidelines. Chest 2013;143:e278S-313S.

4. Wang X, Yan S, Phan K, et al. Mediastinal lymphadenectomy fulfilling NCCN criteria may improve the outcome of clinical N0-1 and pathological N2 non- 
small cell lung cancer. J Thorac Dis 2016;8:342-9.

5. Liu QH, Arias S, Wang KP. International association for the study of lung cancer map, Wang lymph node map and rapid on-site evaluation in transbronchial needle aspiration. J Thorac Dis 2016;8:E869-74.

6. Rusch VW, Asamura H, Watanabe H, et al. The IASLC lung cancer staging project: a proposal for a new international lymph node map in the forthcoming seventh edition of the TNM classification for lung cancer. J Thorac Oncol 2009;4:568-77.

7. Osarogiagbon RU, Eke R, Sareen S, et al. The impact of a novel lung gross dissection protocol on intrapulmonary lymph node retrieval from lung cancer resection specimens. Ann Diagn Pathol 2014;18:220-6.

8. Ramirez RA, Wang CG, Miller LE, et al. Incomplete intrapulmonary lymph node retrieval after routine pathologic examination of resected lung cancer. J Clin Oncol 2012;30:2823-8.

9. Wang X, Yan S, Lv C, et al. Impact of Omission of Intrapulmonary Lymph Node Retrieval on Outcome Evaluation of Lung Cancer Patients Without Lymph Node Metastasis: A Propensity Score Matching Analysis.
Clin Lung Cancer 2017. [Epub ahead of print].

10. Osarogiagbon RU, Hilsenbeck HL, Sales EW, et al. Improving the pathologic evaluation of lung cancer resection specimens. Transl Lung Cancer Res 2015;4:432-7.

11. Smeltzer MP, Faris N, Yu X, et al. Missed Intrapulmonary Lymph Node Metastasis and Survival After Resection of Non-Small Cell Lung Cancer. Ann Thorac Surg 2016;102:448-53.

12. Osarogiagbon RU, Decker PA, Ballman K, et al. Survival Implications of Variation in the Thoroughness of Pathologic Lymph Node Examination in American College of Surgeons Oncology Group Z0030 (Alliance). Ann Thorac Surg 2016;102:363-9.

13. Darling GE, Allen MS, Decker PA, et al. Randomized trial of mediastinal lymph node sampling versus complete lymphadenectomy during pulmonary resection in the patient with N0 or N1 (less than hilar) non-small cell carcinoma: results of the American College of Surgery Oncology Group Z0030 Trial. J Thorac Cardiovasc Surg 2011;141:662-70.
Cite this article as: Tantraworasin A, Taioli E, Siwachat S, Saeteng S. Role of intrapulmonary lymph node retrieval for pathological examination in resectable non-small cell lung cancer. J Thorac Dis 2017;9(11):4280-4282. doi: 10.21037/ jtd.2017.10.118 